\title{
Datos biométricos de cinco especies de murciélagos (Mammalia: Chiroptera) de la Región de Murcia (SE España)
}

\author{
Fulgencio Lisón \\ Departamento de Ecología e Hidrología, Universidad de Murcia, 30100 Campus de Espinardo, Murcia.
}

\begin{abstract}
Resumen
Correspondencia

F. Lisón

E-mail: lison@um.es

Recibido: 1 enero 2012

Aceptado: 19 julio 2012

Publicado on-line: 27 julio 2012

En los murciélagos, las medidas alares son elementos clave para identificar las especies y tienen serias implicaciones en su ecología. No obstante, dichas medidas pueden sufrir variaciones geográficas en el rango de distribución de las especies y en otros casos, puede existir un marcado dimorfismo sexual. En este trabajo se aportan las medidas alares de 5 especies de murciélagos de la Región de Murcia, con un total de 312 individuos analizados. Los resultados confirman que los individuos de nuestra región tienen medidas biométricas dentro del rango de variación de la especie, aunque para algunos valores se ha encontrado que los individuos de Murcia difieren en sus medidas de otros individuos ibéricos. Por otro lado, los resultados confirman la presencia de dimorfismo sexual para las medidas alares en Miniopterus schreibersii. Este trabajo resalta la necesidad de tener un mayor número de datos sobre biometría de los murciélagos ibéricos.
\end{abstract}

Palabras clave: Biometría, Rhinolophus ferrumequinum, Myotis spp., Miniopterus schreibersii, Plecotus austriacus.

\begin{abstract}
Biometric data of five bats species (Mammalia: Chiroptera) of the Region of Murcia (SE Spain)

For bats, wing measurements are key elements for the identification of species and they have serious implications in the ecology of species. However, these measurements can suffer geographical variations inside the distribution of a species and, in other cases, sexual dimorphism happens. In this study, I am contributing with the wing measurement of five bats species on the Region of Murcia (SE, Spain), analysing a total amount of 312 individuals. Theses results confirm that the biometric measurements of our region's bats are within the variation species range, although there are differences in some measurements respect other Iberian bats. In the other hand, my results show the presence of sexual size dimorphism for wing measurements in Miniopterus schreibersii. It is necessary to have more data of biometry of Iberia bats.
\end{abstract}

Key words: Biometry, Rhinolophus ferrumequinum, Myotis spp., Miniopterus schreibersii, Plecotus austriacus.

\section{Introducción}

En los murciélagos, las medidas de las extremidades delanteras tienen valor diagnóstico para la identificación de las especies, ya que la longitud y la anchura del ala tienen importantes implicaciones en la aerodinámica de estos mamíferos voladores y por lo tanto, en muchos aspectos ecológi- 
cos (Norberg \& Rayner 1987, Bogdanowicz et al. 1999, Salsamendi et al. 2005, Dietz et al. 2006, 2009). Además, se ha observado que dentro de una misma especie de murciélago, pueden existir variaciones geográficas (ej. Paz 1995, Arlettaz et al. 1997, Albayrak \& Coşkun 2000, Bornholdt et al. 2008, Furman et al. 2011) e incluso un dimorfismo sexual acusado (Benda 1994).

En este trabajo, se aportan datos biométricos de 5 especies de murciélagos presentes en la Región de Murcia (SE España) que constituyen una valiosa información sobre la morfología de estos mamíferos en la Península Ibérica. También se ha analizado la presencia de dimorfismo sexual en la población del murciélago de cueva Miniopterus schreibersii (Kuhl, 1817).

\section{Material y métodos}

El área de estudio se encuentra en la Región de Murcia, situada en el sureste de la Península Ibérica y con una extensión de $11.313 \mathrm{~km}^{2}$. El clima es típicamente mediterráneo, con temperatura media anual superior a $14^{\circ} \mathrm{C}$ y precipitación media anual de $375 \mathrm{~mm}$.

Los animales fueron capturados entre 2005 y 2010. Las capturas se hicieron durante el día en los refugios, cogiendo a los individuos con la mano o bien por la noche mediante trampas en arpa o redes japonesas. Los animales capturados fueron metidos en bolsas individuales. Posteriormente, los animales fueron sexados e identificados a nivel de especie usando claves de identificación para los murciélagos europeos (Dietz \& von Helversen 2004). Todas las capturas contaron con la correspondiente autorización de la Comunidad Autónoma de la Región de Murcia.

Para los individuos capturados, se tomaron una serie de medidas estándar con un calibre (precisión $\pm 0,1 \mathrm{~mm}$ ) y fueron pesados con una balanza electrónica (precisión $\pm 0,1 \mathrm{~g}$ ). Las medidas alares tomadas fueron: longitud del antebrazo incluyendo la muñeca (FA), longitud del quinto dedo excluyendo la muñeca (D5), longitud del tercer dedo excluyendo la muñeca (D3); además se ha medido longitud entre el canino y el tercer molar superiores $\left(\mathrm{CM}^{3}\right)$. Estas medidas se consideran básicas para la correcta identificación de las especies (Dietz \& von Helversen, 2004) y las primeras proporcionan una descripción simple de las características del ala (Findley et al. 1972, Norberg \&
Rayner 1987, Blood \& McFarlane 1988, Fenton 1990, Dietz et al. 2006). De esta forma, FA es una medida del tamaño del murciélago; D5 refleja la anchura del ala (valores altos son característicos de murciélagos con un vuelo lento, maniobrable y con una carga alar baja); D3 refleja la longitud del ala (valores altos son característicos de los murciélagos con un vuelo rápido y directo); $\mathrm{CM}^{3}$ es una medida importante para la identificación correcta de las especies de los géneros Myotis (Kaup, 1829) y Plecotus (Geoffroy, 1818) (Dietz \& Helversen 2004, Dietz et al. 2009). Además, para Rhinolophus ferrumequinum (Schreber, 1774) se aportan datos de la primera y segunda falange del cuarto dedo (P4.1 y P4.2) y la longitud del primer dedo (D1) que es importante para identificar a las especies del género Plecotus (Dietz \& Helversen 2004, Dietz et al. 2009). Para el estudio únicamente se han empleado individuos adultos y se determinó la edad mediante el grado de osificación de las placas de crecimiento de la epífisis de las falanges de los dedos (Dietz \& von Helversen 2004, Dietz et al. 2009).

Para la especie con un mayor número de individuos (M. schreibersii) se ha realizado un análisis estadístico para determinar la existencia de dimorfismo sexual en las medidas alares (FA, D5 y D3). Para analizar las diferencias entre los sexos, se ha empleado un análisis multivariante de la varianza (MANOVA, test de lambda de Wilks). Adicionalmente, la existencia de diferencias significativas para cada una de las variables consideradas fue analizada mediante tests de ANOVA univariantes. Los análisis fueron realizados con el paquete estadístico R (Maindonald \& Braun, 2010) que se distribuye gratuitamente bajo licencia GNU y está disponible en la URL http://www.rproject.org/.

\section{Resultados}

\section{Biometría de las especies estudiadas}

En total, se capturaron 312 individuos de las cinco especies de murciélagos, siendo el murciélago de cueva, M. schreibersii, la especie con mayor número de individuos analizados $(67 \%)$ y el orejudo gris, Plecotus austriacus (Fischer, 1829), la especie con un menor número de individuos estudiados $(4,5 \%)$. En la Tabla 1 se recoge las medidas biométricas para las cinco especies de murciélagos. En general, las especies murcianas se encuen- 
tran dentro del rango de valores para Europa y el norte de África. Sin embargo, para algunos valores, se ha encontrado que los resultados obtenidos para Murcia son diferentes a los encontrados para la misma especie en otras regiones europeas o de la Península Ibérica.

\section{Dimorfismo sexual en $M$. schreibersii}

Para M. schreibersii (Tabla 2) se ha encuentrado diferencias significativas entre machos y hembras $(\lambda$ Wilks $=0,94167$, d.f. $=205, P<0,01)$, pero sólo para la longitud del antebrazo y la longitud del tercer dedo. En este caso, las hembras son ligeramente mayores que los machos.

\section{Discusión}

Las medidas alares de los murciélagos son consideradas esenciales para el estudio de los mismos, ya que estas medidas tienen valor diagnóstico en la identificación correcta de la especie (Dietz \& von Helversen 2004, Dietz et al. 2009). Además, numerosos estudios han demostrado que dichas medidas biométricas pueden sufrir variaciones geográficas (Albayrak \& Coşkun 2000, Bornholdt et al. 2008), pueden revelar la existencia de un dimorfismo sexual (Benda 1994, Lindenfors et al. 2007) y pueden estar influidas por las condiciones ambientales durante el desarrollo del individuo (Paz 1986; Zahn et al. 2006; Dietz et al. 2007). Por otro lado, la longitud y anchura de las alas tiene importantes implicaciones en la aerodinámica de los individuos (Findley et al. 1972, Norberg \& Rayner 1987, Blood \& McFarlane 1988, Fenton 1990) y estos datos pueden contribuir a explicar cuestiones ecológicas para especies muy similares (Norberg \& Rayner 1987; Bogdanowicz et al. 1999; Salsamendi et al. 2005; Dietz et al. 2006).

En este trabajo, se aportan datos biométricos de cinco especies de murciélagos de la Región de Murcia, un grupo de mamíferos poco conocido actualmente y que se encuentra muy amenazado (Lisón et al. 2010, 2011). Los datos biométricos para las especies murcianas se encuentran dentro del rango descrito para las especies consideradas, aunque se observan algunas variaciones para las poblaciones murcianas.

\begin{tabular}{|c|c|c|c|c|c|c|c|c|c|}
\hline Especie & $\begin{array}{c}\mathbf{n} \\
\left(\partial^{\prime} ; \circ+q\right)\end{array}$ & FA & D5 & D3 & D1 & P4.1 & P4.2 & $\mathrm{CM}^{3}$ & Peso \\
\hline $\begin{array}{l}\text { Rhinolophus } \\
\text { ferrumequinum }\end{array}$ & $\begin{array}{c}41 \\
(19 ; 22)\end{array}$ & $\begin{array}{c}55,2 \pm 1,4 \\
(52,5-58,5)\end{array}$ & $\begin{array}{c}65,6 \pm 3,9 \\
(53,0-71,9)\end{array}$ & $\begin{array}{c}75,8 \pm 4,1 \\
(64,2-82,9)\end{array}$ & & $\begin{array}{l}10,2 \pm 0,7 \\
(8,9-12,0)\end{array}$ & $\begin{array}{c}18,8 \pm 0,8 \\
(17,3-21,4)\end{array}$ & & $\begin{array}{c}16,8 \pm 1,9 \\
(12,5-20,3)\end{array}$ \\
\hline Myotis blythii & $\begin{array}{c}20 \\
(16 ; 4)\end{array}$ & $\begin{array}{c}59,5 \pm 3,1 \\
(52,2-69,3)\end{array}$ & $\begin{array}{c}75,1 \pm 2,6 \\
(67,8-78,4)\end{array}$ & $\begin{array}{c}90,7 \pm 3,2 \\
(82,1-94,5)\end{array}$ & & & & $\begin{array}{l}8,8 \pm 0,2 \\
(8,3-9,1)\end{array}$ & $\begin{array}{c}23,0 \pm 2,0 \\
(19,4-28,1)\end{array}$ \\
\hline $\begin{array}{c}\text { Myotis } \\
\text { capaccinii }\end{array}$ & $\begin{array}{c}28 \\
(15 ; 13)\end{array}$ & $\begin{array}{c}40,6 \pm 1,0 \\
(39,0-42,7)\end{array}$ & $\begin{array}{c}51,2 \pm 1,6 \\
(47,0-54,0)\end{array}$ & $\begin{array}{c}64,1 \pm 2,4 \\
(59,1-69,5)\end{array}$ & & & & & $\begin{array}{c}9,4 \pm 1,1 \\
(7,4-11,8)\end{array}$ \\
\hline $\begin{array}{l}\text { Miniopterus } \\
\text { schreibersii }\end{array}$ & $\begin{array}{c}209 \\
(61 ; 148)\end{array}$ & $\begin{array}{c}44,9 \pm 0,8 \\
(42,9-46,9)\end{array}$ & $\begin{array}{c}51,3 \pm 1,4 \\
(44,5-54,7)\end{array}$ & $\begin{array}{c}80,5 \pm 2,4 \\
(72,8-85,8)\end{array}$ & & & & & $\begin{array}{l}12,3 \pm 1,3 \\
(9,8-17,1)\end{array}$ \\
\hline $\begin{array}{l}\text { Plecotus } \\
\text { austriacus }\end{array}$ & $\begin{array}{c}14 \\
(10 ; 4)\end{array}$ & $\begin{array}{c}40,7 \pm 1,0 \\
(39,5-42,4)\end{array}$ & $\begin{array}{c}53,2 \pm 1,6 \\
(50,0-55,5)\end{array}$ & $\begin{array}{c}67,0 \pm 3,5 \\
(63,0-77,3)\end{array}$ & $\begin{array}{l}5,6 \pm 0,6 \\
(4,5-6,2)\end{array}$ & & & $\begin{array}{l}6,0 \pm 0,1 \\
(5,8-6,2)\end{array}$ & $\begin{array}{c}9,1 \pm 0,6 \\
(8,3-10,1)\end{array}$ \\
\hline
\end{tabular}

Tabla 1. Datos biométricos para cada especie en la Región de Murcia. Se indica el número total de individuos medidos y su sexo. Para cada medida se indica la media \pm SD y su rango. Todas las medidas están en milímetros, excepto el peso que se indica en gramos.

Table 1. Biometrics data for each bat species of the Region of Murcia. The total number of individuals measured and their sex is indicated. All measurements are indicated as mean $\pm \mathrm{SD}$ and their range values. All measurements are in millimeters, except the weight which is expressed in grams.

\begin{tabular}{cccccc}
\hline & & Machos $(\mathrm{n}=61)$ & Hembras $(\mathrm{n}=148)$ & Valor $\mathrm{F}$ & $\mathrm{P}$ \\
\hline \multirow{3}{*}{ Miniopterus schreibersii } & FA & $44.7 \pm 0.8(42.8-46.7)$ & $45.0 \pm 0.7(43.1-46.9)$ & 8.5837 & $* *$ \\
& D5 & $51.1 \pm 1.5(44.5-53.5)$ & $51.3 \pm 1.3(47.7-54.7)$ & 1.2018 & $\mathrm{~ns}$ \\
& D3 & $79.8 \pm 2.4(73.9-84.5)$ & $80.8 \pm 2.3(72.8-85.8)$ & 8.6034 & $* *$ \\
\hline
\end{tabular}

Significado de los códigos: ${ }^{* *} \mathrm{P}<0.001 ;{ }^{* *} \mathrm{P}<0.01 ;{ }^{*} \mathrm{P}<0.05$ y $\mathrm{ns}=$ no significativo

Tabla 2. Medidas alares para ambos sexos en adultos de Miniopterus schreibersii para el sureste de la Península Ibérica. El valor (media \pm SD), rango y análisis estadístico (ANOVA) para la longitud del antebrazo (FA), longitud del quinto dedo (D5) y longitud del tercer dedo (D3).

Table 2. External wing measurements for both sexes of adults of Miniopterus schreibersii from South-Eastern Iberia. The value (mean \pm SD), range and statistics analysis (ANOVA) for forearm length (FA), length of $5^{\text {th }}$ finger (D5) and length of $3^{\text {rd }}$ finger (D3). 
Para el murciélago grande de herradura, $R$. $f e$ rrumequinum, las medidas del antebrazo y de los dedos son ligeramente inferiores a las que encontramos para la especie en la cuenca mediterránea, especialmente la longitud del tercer dedo (D3 = 79-94 mm; Dietz et al. 2009). No obstante, las medidas de las falanges del cuarto dedo se mantienen en los rangos europeos. La medidas de antebrazo para el País Vasco $(\mathrm{FA}=54,9 \pm 1,6$; Aihartza 2005) y para Navarra $(\mathrm{FA}=55,2 \pm 1,6$; Alcalde 1995) muestra que el tamaño es muy similar en el norte y sur de España. Sin embargo, los individuos de Murcia tienen un menor peso (Peso País Vasco $=18,2 \pm 2,2$; Peso Navarra $=18,2$ $\pm 2,0)$. Esto puede estar motivado por factores ambientales o geográficos, ya que se ha comprobado en la región paleártica se produce un incremento gradual de tamaño en las dimensiones externas desde el sudoeste hacia el noreste, con los especímenes del norte de África siendo los de menor tamaño (Paz 1995).

Para el murciélago ratonero mediano, Myotis blythii (Tomes, 1857), los valores medios de los individuos de Murcia se encuentran dentro de los parámetros normales para la especie en su distribución europea (Dietz et al. 2009).

Para el murciélago ratonero patudo, Myotis capaccinii (Bonaparte, 1837), los valores del antebrazo y del quinto dedo se ajustan bastante al rango de la especie, sin embargo, los valores del tercer dedo son más bajos que los que se encuentran para la especie en otras zonas de la cuenca mediterránea (D3=64-71 mm; Dietz et al. 2009).

Los resultados para $M$. schreibersii indican que los individuos de Murcia se encuentran dentro del rango de la especie, aunque muy cercanos al límite inferior. Cuando comparamos las medidas de antebrazo en País Vasco (FA=45,4 $\pm 0,8$; Aihartza 2005) y Navarra $(\mathrm{FA}=45,9 \pm 0,6$; Alcalde 1995), los individuos de Murcia son ligeramente más pequeños (Tabla 1). En cuanto al peso de los individuos, también encontramos que los individuos de nuestra área de estudio tienen un peso inferior (Peso País Vasco=13,1 $\pm 1,7$; Peso Navarra $=14,4 \pm 1,6)$. No obstante, las diferencias en el peso deben ser consideradas con cuidado, ya que la especie muestra una acusada variación en el peso según la estación (Lisón 2012). Por otro lado, la presencia de dimorfismo en las medidas alares hasta ahora no era muy conocida y su existencia en Murcia puede deberse a variaciones geográfi- cas de la especie (Albayrak \& Coşkun 2000) que hasta ahora no se habían detectado o bien a aislamiento genético de las poblaciones del sureste peninsular como ocurre en Portugal (Ramos-Pereira et al. 2009).

Por último, los valores para $P$. austriacus se ajustan a las medidas realizadas en las poblaciones europeas, aunque el número de individuos capturados en nuestra zona es muy bajo. Las medidas del antebrazo son muy similares a las encontradas en Navarra ( $\mathrm{FA}=40,6 \pm 0,9$; Alcalde 1995) y otras zonas peninsulares $(\mathrm{FA}=40,5 \pm 1,4$; $\mathrm{Paz}$ 1994). Pero inferiores a las encontradas en el País Vasco $(F A=41,8 \pm 0,8$; Aihartza 2005). En cuanto al peso, los individuos de Murcia tienen un peso similar al encontrado en País Vasco (Peso= $9,2 \pm 0,7$; Aihartza 2005) pero menor que el encontrado en Navarra (Peso $=9,9 \pm 2,2$; Alcalde 1995).

Los datos biométricos de la morfología alar de los murciélagos se consideran actualmente como una herramienta esencial para el desarrollo de diferentes investigaciones sobre ecología de murciélagos (Bogdanowicz et al. 1999, Salsamendi et al. 2005, Dietz et al. 2006, 2007, 2009), especialmente si se tratan de criptoespecies (Ibáñez et al. 2006). Sin embargo, la información disponible para los individuos ibéricos es escasa, limitándose a unas pocas medidas, especialmente la longitud del antebrazo (Ibáñez 1998; Fernández 2002; Palomo et al. 2007) o datos de unos pocos individuos de la misma especie (Lisón et al. 2005; Salsamendi et al. 2005; Trujillo \& García 2009). Curiosamente, es habitual que los investigadores tomen estas medidas para la identificación de las especies (Alcalde et al. 2008, Trujillo \& García 2009, Flaquer et al. 2010, Lisón et al. 2010). El conocimiento de datos biométricos en los murciélagos puede poner de manifiesto diferencias latitudinales, geográficas, ambientales o genéticas que son claves para la conservación de estos mamíferos.

\section{Agradecimientos}

Agradezco a los técnicos y al personal de la red de Espacios Naturales Protegidos de la Región de Murcia la localización de los refugios y las facilidades prestadas para desarrollar los diferentes trabajos, especialmente a Emilio Aledo y Néstor D. Yelo. Agradezco la generosidad de Joxerra Aihartza y Juan Tomas Alcalde, por dejarme indagar en 
la base de datos de sus tesis. Agradezco el apoyo y la paciencia de José F. Calvo y sus valiosas enseñanzas en ecología. También agradezco la valiosa compañía de la gente que me ha ayudado en las mediciones: José A. López, Severiano Jiménez, Mario León, Salvador Rubio, Francisco Almansa, Félix Picazo, Julián Picazo y Carlos GonzálezRevelles. Agradezco los valiosos comentarios y la cortesía de Javier Juste. Agradezco los comentarios de los dos revisores anónimos que han contribuido a mejorar sustancialmente el manuscrito original. Este estudio no se hubiera realizado sin el apoyo constante y el sacrificio personal de Ángeles Haz.

\section{Referencias}

Aihartza JR. 2005. Quirópteros de Araba, Bizkaia y Gipuzkoa: distribución, ecología y conservación. Tesis Doctoral. Universidad del País Vasco. 346 pp.

Albayrak I. \& Coşkun Ş. 2000. Geographic variations and taxonomic status of Miniopterus schreibersii (Kuhl, 1819) in Turkey (Chiroptera: Vespertilionidae). Turkey Journal of Zoology 24: 125-133.

Alcalde JT. 1995. Distribución y fenología de los quirópteros en Navarra. Tesis Doctoral. Universidad de Navarra. 430 pp.

Alcalde JT, Trujillo D, Artázcoz A \& Agirre-Mendi PT. 2008. Distribución y estado de conservación de los quirópteros en Aragón. Graellsia 64(1): 3-16.

Arlettaz R, Ruedi M, Ibáñez C, Palmeirim J \& Hausser J. 1997. A new perspective on the zoogeography of the sibling mouse-eared bat species Myotis myotis and Myotis blythii: morphological, genetical and ecological evidence. Journal of Zoology 242: 45-62.

Benda P. 1994. Biometrics of Myotis myotis and Myotis blythii: age variation and sexual dimorphism. Folia Zoologica, 43(4): 297-306.

Blood BR \& McFarlane DA. 1988. A new method for calculating the wing area of bats. Mammalia 52: 600603.

Bogdanowicz W, Fenton MB \& Daleszczyk K. 1999. The relationship between echolocation calls, morphology and diet in insectivorous bats. Journal of Zoology 247: 381-393.

Bornholdt R, Oliveira LR \& Fabián ME. 2008. Sexual size dimorphism in Myotis nigricans (Schinz, 1821) (Chiroptera: Vespertilionidae) from south Brazil. Brazilian Journal of Biology 68(4): 897-904.

Dietz C \& von Helversen O. 2004. Identification key to the bats of Europe. Electronical publication, version 1.0. Distribuido por el autor. Disponible en internet en <http://www.fledermaus-dietz.de/publications/ publications.html> [accedido el 15 diciembre 2008].

Dietz C, Dietz I \& Siemers BM. 2006. Wing measurement variations in the five European horseshoe bat species (Chiroptera: Rhinolophidae). Journal of Mammalogy 87(6): 1241-1251.
Dietz C, Dietz I \& Siemers BM. 2007. Growth of horseshoe bats (Chiroptera: Rhinolophidae) in temperate continental conditions and the influence of climate. Mammalian Biology 72: 129-144.

Dietz C, von Helversen O \& Nill D. 2009. Bats of Britain, Europe \& Northerwest Africa. A\&C Black, London.

Fenton MB. 1990. The foraging behaviour and ecology of animal-eating bats. Canadian Journal of Zoology 68: 411-422.

Fernández J. 2002. Los murciélagos en Castilla y León. Atlas de distribución y tamaño de las poblaciones. Consejería de Medio Ambiente. Junta de Castilla y León.

Findley JS, Studier EH \& Wilson DE. 1972. Morphological properties of bat wings. Journal of Mammalogy 53: 429-444.

Flaquer C, Puig X, Fàbregas E, Guixé D, Torre I, Ràfols RG, Páramo F, Camprodon J, Cumplido JM, RuízJarillo $R$, Baucells $A L$, Freixas $L$ \& Arrizabalaga $A$. 2010. Revisión y aportación de datos sobre quirópteros de Catalunya: Propuesta de Lista Roja. Galemys 22 (1): 29-61.

Furman A, Bachanek J, Postawa T \& Çoraman E. 2011. Morphometric variation and genetic diversity of the lesser and greater mouse-eared bats (Chiroptera: Vespertilionidae) in Thrace and Anatolia. Acta Chiropterologica, 13(2): 291-298.

Ibáñez C, García-Mudarra JL, Ruedi M, Stadelmann B \& Juste J. 2006. The Iberian contribution to cryptic diversity in European bats. Acta Chiropterologica 8(2): 277-297.

Ibáñez C. 1998. Orden Quirópteros. En Mamíferos de España; Volumen I: Insectívoros, Quirópteros, Primates y Carnívoros de la Península Ibérica, Baleares y Canarias (Blanco JC, ed). Editorial Geoplaneta, pp: $114-218$.

Lindenfors P, Gittleman JL \& Jones KE. 2007. Sexual size dimorphism in mammals. En Sex, Size, and Gender Roles: Evolutionary Studies of Sexual Size Dimorphism. (Fairbairn DJ, Blanckenhorn WU \& Székely T, eds). Oxford University Press, pp. 16-26.

Lisón F. 2012. Murciélago de cueva - Miniopterus schreibersii. En Enciclopedia Virtual de los Vertebrados Españoles (Salvador A \& Cassinello J, eds). Museo Nacional de Ciencias Naturales, Madrid. Disponible en internet en <http://www.vertebradosibericos.org/> [accedido el 01 junio 2012].

Lisón F, Aledo E \& Calvo JF. 2011. Los murciélagos (Mammalia: Chiroptera) de la Región de Murcia (SE España): distribución y estado de conservación. Anales de Biología 33: 79-92.

Lisón F, Yelo ND, Balsalobre M \& Calvo JF. 2005. Primeros datos sobre el murciélago de Nathusius Pipistrellus nathusii (Keyserling y Blasius, 1839) en la Región de Murcia. Galemys 17(1-2): 47-52.

Lisón F, Yelo ND, Haz Á \& Calvo JF. 2010. Contribución al conocimiento de la distribución de la fauna quiropterológica de la Región de Murcia. Galemys 22 (1): $11-28$.

Maindonald J \& Braun WJ. 2010. Data analysis and graphics using R. 3rd edn. Cambridge University Press, Cambridge. 
Norberg UM \& Rayner JMV. 1987. Ecological morphology and flight in bats: wing adaptations, flight performance, foraging strategy and echolocation. Philosophical Transactions of the Royal Society of London, B. Biological Sciences 316: 335-427.

Palomo LJ, Gisbert J \& Blanco JC. 2007. Atlas y Libro Rojo de los Mamíferos Terrestres de España. Dirección General de la Biodiversidad-SECEM-SECEMU, Madrid.

Paz O. 1986. Age estimation and postnatal growth of the Greater mouse bat Myotis myotis (Borkhausen, 1797) in Guadalajara, Spain. Mammalia, 50: 243252.

Paz O. 1994. Systematic position of Plecotus (Geoffroy, 1818) from the Iberian Peninsula (Mammalia: Chiroptera). Mamalia 58(3): 423-432.

Paz O. 1995. Geographic variation of the Greater horseshoe bat (Rhinolophus ferrumequinum) in the westhalf of the Paleartic region. Myotis, 32: 33-44
Ramos-Pereira JM, Salgueiro P, Rodrigues L, Coelho MM \& Palmeirim JM. 2009. Population structure of a cave-dwelling bat, Miniopterus schreibersii: does it reflect history and social organization? Journal of Heredity, 100(5): 533-544.

Salsamendi E, Aihartza JR, Goiti U, Almenar D \& Garín I. 2005. Echolocation calls and morphology in the Mehelyi's (Rhinolophus mehelyi) and Mediterranean (R. euryale) horseshoe bats: implications for resource partitioning. Hystrix Italian Journal of Mammalogy 16(2): 149-158.

Trujillo D \& García D. 2009. Primera cita del murciélago de Nathusius Pipistrellus nathusii (Keyserling y Blasius, 1839) para las Islas Baleares. Galemys 21(2): 39-46.

Zahn A, Rottenwallner A \& Güttinger R. 2006. Population density of the Greater mouse-eared bat (Myotis myotis), local diet composition and availability of foraging habitats. Journal of Zoology 269: 486-496. 